doi: http://dx.doi.org/10.5892/ruvrv.2013.111.7883

\title{
Avaliação da adequada indicação de leite artificial em recém-nascidos em uma maternidade de referência de Minas Gerais
}

\begin{abstract}
Amanda S. CERÁVOLO', Enilda B. ARAÚJO' ${ }^{1}$, Acadêmica de Medicina Maria Isabel S. CARVALHO ${ }^{1}$, Acadêmica de Medicina Weslaine O. MAIA ${ }^{1}$, Acadêmica de Medicina Edson B. SOUZA², Médico Ginecologista e Obstetra Soraya M. C. GROSSMANN ${ }^{1}$, Professora, Mestre, Doutora

${ }^{1}$ Universidade Vale do Rio Verde - UNINCOR - Belo Horizonte - MG*

${ }^{2}$ Hospital Sofia Feldman - Belo Horizonte - MG

Recebido em: 10/05/2013 - Aprovado em: 10/07/2013 - Disponibilizado em: 15/08/2013

\section{Resumo}

Este estudo teve como objetivo verificar a prevalência e identificar justificativas alegadas para o uso da suplementação ao aleitamento materno em recém-nascidos de um alojamento conjunto. Foi um estudo transversal, quantitativo e retrospectivo. A amostra foi composta por 39 recém-nascidos de um Hospital Amigo da Criança de Belo Horizonte, Brasil, que fizeram uso de suplemento lácteo no alojamento conjunto. As justificativas alegadas para uso de suplemento foram classificadas como aceitáveis ou não segundo a OMS. Dos 1299 nascidos vivos, apenas 57 tiveram solicitação no lactário para utilização de leite artificial e destes teve-se acesso a 39 prontuários. As mães estudadas estavam dentro da faixa etária de 21 a 35 anos, casadas, possuíam ensino fundamental e realizaram 6 ou mais consultas de pré-natal. Os recém-nascidos estudados nasceram em sua maioria a termo, com peso adequado para idade gestacional e com ápgar de primeiro e quinto minuto, maior ou igual a 8 e iniciaram o uso de suplemento lácteo em sua maioria nas primeiras 12 horas. A prevalência de uso de suplemento foi de 4,54\%, sendo a principal indicação a hipoglicemia. Conclui-se que, as poucas indicações prescritas para o uso de fórmula láctea pelo HAC avaliado foram adequadas de acordo com o que a OMS preconiza, assim percebe-se que a instituição estudada atende às políticas defendidas pela IHAC.
\end{abstract}

Palavras chaves: aleitamento; leite artificial; fórmula; recém-nascido.

\section{Abstract}

This study aimed to determine the prevalence and reasons for the use of supplementation of breastfeeding in infants born. A cross sectional study, quantitative and retrospective study, included a sample of 39 newborns at Hospital of Belo Horizonte, Brazil, who used supplement in milk rooms. Reasons for supplement use were classified as acceptable or not according to WHO. Of 1299 live births, only 57 were in lactary request for use of artificial milk, and these had access to 39 records. The women studied were in the age group 21-35 years, married, had elementary school and performed six or more prenatal consultations. The newborn infants studied were born mostly at full term with appropriate weight for gestational age and Apgar score at first and fifth minutes, greater than or equal to 8 and started 
using milk supplementation mostly in the first 12 hours. The prevalence of supplement use was $4.54 \%$, being the main indication hypoglycemia. We conclude that the low prescribed for the use of infant formula by Hospital evaluated were appropriate according to the WHO recommends that, so it is clear that the institution meets the studied policies advocated.

Keywords: breastfeeding, formula feeding, formula, baby

\section{Introdução}

O leite materno é um alimento completo para o recém-nascido, pois fornece componentes nutricionais e imunológicos que proporcionam um efeito protetor contra inúmeras doenças ${ }^{1-3}$. Devido à imaturidade do sistema imune o recém-nascido é mais vulnerável às infecções ${ }^{4-6}$. $\mathrm{O}$ colostro, o primeiro leite produzido pela mãe que possui uma importância muito grande, apesar de pequeno volume é apropriado para o tamanho do estômago do recém-nascido, contribuindo para que o este aprenda a coordenar a sucção, deglutição e respiração ${ }^{7-10}$. Além disso, a mãe que oferece o aleitamento materno exclusivo, mantém uma maior proximidade corporal e emocional que em muito contribui para a relação entre mãe e filho ${ }^{11,12}$. O aleitamento materno gera repercussões positivas para a sociedade, reduzindo os índices de morbidade e mortalidade infantil ${ }^{13}$. Portanto, é necessário que o pediatra tenha motivos relevantes ao substituir o leite materno pelo uso de fórmula láctea $^{14,15}$. O leite humano ordenhado é a primeira opção para alimentação suplementar oferecido aos recém-nascidos quando o leite materno não pode ser sugado diretamente da mama $^{7}$.
O uso indiscriminado de suplementos na maternidade hospitalar aumenta o risco de desmame, já que pode levar as mães a continuarem o seu uso em domicílio ${ }^{8}$. Assim a produção de leite, que aumenta progressivamente durante a amamentação, pode sofrer uma diminuição juntamente com a redução da frequiência das mamadas ${ }^{9}$. Além disso, pode diminuir a confiança da mãe sobre sua capacidade de atender às necessidades nutricionais do seu bebê $\hat{e}^{7}$.

Estudos que investigam o uso de fórmula em substituição ao leite materno são de suma importância, para que haja uma mudança de conduta na prescrição indiscriminada do uso de fórmulas para recém-nascidos. Dessa forma, objetivou-se com o presente estudo avaliar a prevalência do uso da suplementação com leites artificiais em recém-nascidos de um Hospital Amigo da Criança, comparando a adequação do seu uso com as recomendações da OMS.

\section{Metodologia}

Este estudo transversal, quantitativo e retrospectivo foi realizado no alojamento conjunto do Hospital Sofia Feldman. Esta instituição possui o título de Hospital Amigo 
da Criança e está localizada no município de Belo Horizonte, MG, sendo um importante centro de referência materno-infantil desta região metropolitana.

O Hospital Sofia Feldman assiste a uma população superior a 400 mil pessoas dos Distritos Sanitários Norte e Nordeste, em Belo Horizonte. Possui 130 leitos: 60 obstétricos, 41 em Unidade de Tratamento Intensivo Neonatal - UTI, 23 em Unidade de Cuidados Intermediários Neonatais - UCI, e 16 de outras clínicas. São realizados cerca de 800 partos ao mês. A assistência maternoinfantil, com todas as internações feitas através da Central de Internações da Secretaria Municipal de Saúde, pelo SUS.

Durante um período de 2 meses, foram levantados os prontuários e as fichas de notificação do uso de substitutos artificiais do leite materno nos recém-nascidos admitidos no alojamento conjunto. Foram incluídos todos os casos de recém-nascidos de termo, ou próximos do termo (34-36 semanas e 6 dias) que tenham recebido suplementação com leite artificial (qualquer volume), durante o período de internação. Foram excluídos os recém-nascidos prematuros admitidos à unidade de terapia intensiva (UTI) ou unidade de cuidados intensivos (UCI) e os bebês readmitidos ao hospital após terem recebido alta hospitalar.

Em relação às indicações do uso de suplementos lácteos, foram consideradas adequadas aquelas que estivessem em conformidade com as recomendações da $\mathrm{OMS}^{10}$, e inadequadas aquelas indicações que não estiveram em conformidade com as recomendações deste órgão.

As razões médicas aceitáveis para uso de substitutos do leite materno, de acordo com a OMS e Unicef $^{16}$, foram: crianças com erros inatos do metabolismo (galactosemia clássica, fenilcetonúria, doença da urina do xarope de bordo); crianças nascidas com peso inferior a $1500 \mathrm{~g}$; crianças nascidas com idade inferior a 32 semanas;crianças com risco de hipoglicemia e crianças filhos de mães diabéticas.

As condições médicas maternas que justificam o uso de amamentação artificial permanente foram: mães HIV positivas; mães com doença grave que a impossibilite de cuidar do seu bebê, como septicemia; mães com herpes simples tipo I; mães em uso de medicamentos sedativos, anticonvulsivantes, opióides e combinações que podem causar efeitos colaterais como tonturas e depressão respiratória; mães em uso excessivo de iodotópica ou iodóforo e mães que estão em processo de quimioterapia citotóxica ${ }^{10}$.

Para a relação hipoglicemia do recémnascido, foi adotado um valor de corte de $40 \mathrm{mg} / \mathrm{ml}$ (quarenta miligramas por mililitros) ${ }^{17}$.

Os casos foram identificados através das Fichas de Solicitação de Fórmula recebidas pelo lactário do hospital, indispensáveis para a dispensa de todo leite artificial oferecido aos recém-nascidos. Todos 
os dados foram coletados por acadêmicas do curso de Medicina da UNINCOR, sob a supervisão da orientadora e co-orientador do estudo. Utilizou-se ficha pré-elaborada para o presente estudo, na qual incluía, além de dados demográficos, aqueles relativos à caracterização da mãe e do recém-nascido e indicações do uso de fórmula láctea. Posteriormente, os dados foram compilados em um banco de dados. Para a análise dos dados quantitativos utilizou-se as freqüências absolutas e relativas e o teste do qui-quadrado com nível de significância de $\mathrm{p} \leq 0,05$ para identificação de diferenças no padrão de utilização de fórmula láctea em recémnascido (RN) na unidade de internação estudada, por meio do software SPSS versão 15.0. Os resultados também foram analisados tendo como parâmetros as diretrizes da Organização Mundial de Saúde e da UNICEF e artigos da literatura científica sobre o tema.

A divulgação dos resultados está sendo realizado por meio de trabalho de conclusão de curso de Medicina, apresentado à Universidade Vale do Rio Verde UNINCOR, com o objetivo de publicação posterior.

Este trabalho foi aprovado pelos comitês de ética em pesquisa do Hospital Sofia Feldman e Universidade Vale do Rio Verde - UNINCOR.

\section{Resultados}

Em 2 meses de coleta no Hospital Amigo da Criança houve um número de 1299 nascidos vivos. Destes, apenas 57 recémnascidos tiveram solicitação no lactário para utilização de leite artificial, o que corresponde a $4,39 \%$ dos neonatos. Do total de 57 solicitações para uso de fórmula láctea, tevese acesso a 39 prontuários para análise dos dados dos recém-nascidos e de suas mães, devido a não disponibilidade dos demais prontuários.

A população estudada é composta por 39 recém-nascidos que tiveram leite artificial prescrito. Os recém-nascidos permaneceram exclusivamente no alojamento conjunto, nasceram em sua maioria a termo (37 semanas a 41 semanas e 6 dias), sendo a menor idade gestacional de 34 semanas e 2 dias e a maior de 41 semanas e 3 dias. Em relação ao peso, predominou-se um valor maior ou igual a 2500 gramas, com média de peso ao nascer de 2934 gramas, sendo o peso mínimo 1545 gramas e o peso máximo 4100 gramas.

As mães incluídas no estudo cujos recém-nascidos fizeram uso de leite artificial, estavam em sua maioria dentro da faixa etária de 21 a 35 anos, eram casadas e possuíam ensino fundamental. Durante a gestação, a maioria das mães realizaram 6 ou mais consultas de pré-natal, a idade gestacional que prevaleceu foi a termo (maior que 37 e menor que 41 semanas e 6 dias), sendo a via de parto 
predominante a via normal, de ordem única, não usando ocitocina, analgesia e antibiótico na maioria dos casos. A maior parte das mães não fazia uso de medicamentos de uso contínuo e não possuíam patologias associadas (Tabela 1).

Tabela 1 - Características das mães dos recém-nascidos (RNs) em uso de fórmula láctea e seus respectivos dados obstétricos em uma unidade de internação, BH-MG, 2011.

\begin{tabular}{|c|c|c|}
\hline Características da mãe e dados obstétricos & $\mathrm{N}=39$ & $(\%)$ \\
\hline \multicolumn{3}{|l|}{ Idade: } \\
\hline$\leq 20$ anos & 10 & 25,64 \\
\hline $21-35$ anos & 25 & 64,10 \\
\hline$\geq 36$ anos & 4 & 10,26 \\
\hline \multicolumn{3}{|l|}{ Estado Civil: } \\
\hline Solteira & 18 & 46,15 \\
\hline Casada & 21 & 53,85 \\
\hline \multicolumn{3}{|l|}{ Escolaridade: } \\
\hline Ensino Fundamental* & 25 & 64,10 \\
\hline Ensino Médio & 14 & 35,90 \\
\hline \multicolumn{3}{|l|}{ Via de parto: } \\
\hline Césarea & 16 & 41,03 \\
\hline Normal & 23 & 58,97 \\
\hline \multicolumn{3}{|l|}{ Número de consultas de pré-natal: } \\
\hline$\leq 5$ & 15 & 38,46 \\
\hline$\geq 6$ & 24 & 61,54 \\
\hline \multicolumn{3}{|l|}{ Uso de medicamentos: } \\
\hline Sim & 12 & 30,77 \\
\hline Não & 27 & 69,23 \\
\hline \multicolumn{3}{|l|}{ Patologias associadas: } \\
\hline Nenhuma & 20 & 51,28 \\
\hline Amniorrexe prematura & 4 & 10,26 \\
\hline Trabalho de parto prematuro & 6 & 15,38 \\
\hline Outras( pré-eclâmpsia, asma, epilepsia, HIV, toxoplasmose, hipertensão) & 9 & 23,08 \\
\hline \multicolumn{3}{|l|}{ Idade Gestacional: } \\
\hline Pré-termo & 13 & 33,33 \\
\hline Termo & 26 & 66,67 \\
\hline \multicolumn{3}{|l|}{ Ordem } \\
\hline Único & 33 & 84,62 \\
\hline Gemelar & 6 & 15,38 \\
\hline \multicolumn{3}{|l|}{ Trabalho de parto } \\
\hline Espontâneo & 35 & 89,74 \\
\hline Induzido & 4 & 10,26 \\
\hline \multicolumn{3}{|l|}{ Ocitocina } \\
\hline Sim & 12 & 30,77 \\
\hline Não & 27 & 69,23 \\
\hline \multicolumn{3}{|l|}{ Analgesia } \\
\hline Sim & 14 & 35,90 \\
\hline Não & 25 & 64,10 \\
\hline Uso de antibiótico & & \\
\hline
\end{tabular}




\begin{tabular}{l|r|r}
\hline Sim & 43,59 & 17 \\
Não & 22 & 56,41 \\
\hline
\end{tabular}

*Ensino fundamental inclui 1 paciente não alfabetizada.

Os recém-nascidos em estudo apresentou má formação congênita. Estes apresentaram ao nascimento em sua maioria Apgar de $1^{\circ}$ e $5^{\circ}$ minuto maior ou igual a 8.0 neonatos iniciaram o uso de suplemento lácteo, em sua maioria, antes das primeiras 12 peso predominante dos neonatos foi maior ou horas de vida (Tabela 2).

igual a 2500 gramas e somente 1 caso

Tabela 2 - Características dos RNs em uso de fórmula láctea em uma unidade de internação, BH-MG, 2011.

\begin{tabular}{l|c|c}
\hline Características do recém-nascido & $\mathrm{N}=50$ & $(\%)$ \\
\hline Apgar de $\mathbf{1}^{\mathbf{0}}$ minuto & & 17,95 \\
$\leq 7$ & 32 & 82,05 \\
$\geq 8$ & & \\
\hline Apgar de 5 $\mathbf{5}^{\mathbf{0}}$ minuto & 1 & 2,56 \\
$\leq 7$ & 38 & 97,44 \\
$\geq 8$ & & \\
\hline Peso ao nascer (g): & 9 & 23,08 \\
1500 a 2500 & 30 & 76,92 \\
$\geq 2500$ & & \\
\hline Hora pós-natal que se iniciou o uso de fórmula & 25 & 64,10 \\
$1-12$ & 10 & 25,64 \\
$13-24$ & 4 & 10,26 \\
$\geq 25$ & & \\
\hline Má formações congênitas & 1 & 2,56 \\
Sim & 38 & 97,44 \\
Não & & \\
\hline
\end{tabular}

A indicação para uso de fórmula láctea em RNs com fatores de risco para glicemia (prematuridade, pequeno para idade gestacional - PIG, grande para idade gestacional - GIG, quadro infeccioso, sofrimento intra-parto), com dificuldade de sucção nas primeiras 48 horas e colostro ausente à expressão manual, foi a mais prevalente dentre as indicações da instituição (Tabela 3). 
Tabela 3 - RNs que fizeram uso de fórmula láctea de acordo com as indicações da unidade de internação onde foi realizado o estudo.

\begin{tabular}{|c|c|c|}
\hline Indicações da unidade de internação & $\mathbf{N}(39)$ & $\%$ \\
\hline \multicolumn{3}{|l|}{ Indicações Absolutas } \\
\hline RN cuja mãe esteja ausente por qualquer motivo; & 4 & 10,26 \\
\hline RN cuja mãe apresente herpes com lesões ativas em mamas; & $\mathbf{0}$ & $\mathbf{0}$ \\
\hline $\begin{array}{l}\text { RN cuja mãe esteja em uso de medicamentos citotóxicos, radioativos, antireoidianos, exceto } \\
\text { propiltiouracil; }\end{array}$ & $\mathbf{0}$ & $\mathbf{0}$ \\
\hline RN cuja mãe seja soropositiva para HIV ou HTLV; & 3 & 7,69 \\
\hline RN com erro inato do metabolismo (galactosemia, fenilcetonúria, doença xarope de bordo). & $\mathbf{0}$ & $\mathbf{0}$ \\
\hline \multicolumn{3}{|l|}{ Indicações Relativas } \\
\hline $\begin{array}{l}\text { RN com quadro de hipoglicemia, confirmado por exame laboratorial, quando os níveis glicêmicos não } \\
\text { respondem ao aleitamento materno ótimo, ou a alimentação com o leite materno; }\end{array}$ & 7 & 17,95 \\
\hline $\begin{array}{l}\text { RN com fatores de risco para glicemia (prematuridade, PIG, GIG, quadro infeccioso, sofrimento intra- } \\
\text { parto), com dificuldade de sucção nas primeiras } 48 \text { horas e colostro ausente à expressão manual; } \\
\text { RN cuja mãe apresente doença grave, na qual o contato pele a pele e/ou a ordenha não sejam possíveis } \\
\text { (mãe inconsciente, ventilação mecânica, ou colostro ausente/insuficiente para necessidades diárias). }\end{array}$ & 23 & $\begin{array}{r}58,97 \\
5,13\end{array}$ \\
\hline
\end{tabular}

Conforme as indicações da OMS para as razões médicas aceitáveis e condições médicas maternas que justifiquem o uso de amamentação artificial permanente, verificouse predominância no quesito recém-nascido com risco para hipoglicemia (Tabela 4).

Tabela 4- Recém-nascidos que fizeram uso de fórmula láctea em unidade de internação, BH-MG, 2011, em conformidade com as razões médicas aceitáveis preconizadas pela OMS.

\begin{tabular}{|c|c|c|}
\hline Indicações para Suplementação Láctea conforme a OMS & $\mathbf{N}(39)$ & $\%$ \\
\hline Razões Médicas aceitáveis & \multirow{7}{*}{30} & \multirow{7}{*}{76,93} \\
\hline Crianças com erros inatos do metabolismo (galactosemia clássica, fenilcetonúria, doença da urina e do & & \\
\hline xarope de bordo); & & \\
\hline Crianças nascidas com peso inferior a 1500 gramas; & & \\
\hline Crianças nascidas com idade inferior a 32 semanas; & & \\
\hline Crianças com risco de hipoglicemia*; & & \\
\hline Crianças filhos de mãe diabética. & & \\
\hline \multicolumn{3}{|l|}{ Condições médicas maternas que justificam o uso } \\
\hline Mães HIV positivas; & 3 & 7,69 \\
\hline Mães com doença grave que a impossibilite de cuidar do seu bebê, como septicemia; & 2 & 5,13 \\
\hline \multicolumn{3}{|l|}{ Mães com herpes simples tipo I; } \\
\hline \multirow{2}{*}{\multicolumn{3}{|c|}{$\begin{array}{l}\text { Mães em uso de medicamentos sedativos, anticonvulsivantes, opióides e combinações que podem } \\
\text { causar efeitos colaterais como tonturas e depressão respiratória; }\end{array}$}} \\
\hline & & \\
\hline \multicolumn{3}{|l|}{ Mães em uso excessivo de iodo tópico ou iodóforo; } \\
\hline Mães que estão em processo de quimioterapia citotóxica. & & \\
\hline
\end{tabular}

*Englobou-se neste item 7 casos de recém-nascidos com hipoglicemia confirmada, sendo que os estes não apresentaram fatores de risco associados para tal. 
Foi encontrada uma significância estatística na relação entre tentativa de ordenha e indicação de fórmula láctea. Na maioria das mães pesquisadas, observou-se que houve tentativa de ordenha, sendo que a indicação predominante foi risco para hipoglicemia

(Tabela

Tabela 5 - Estratificação de tentativa de ordenha correlacionada com a indicação do uso de fórmula láctea.

\begin{tabular}{|c|c|c|c|c|c|c|c|c|}
\hline & & \multicolumn{5}{|c|}{ Indicação da fórmula } & \multirow[t]{2}{*}{ Total } & \multirow{2}{*}{$\begin{array}{c}\text { Valor } \\
p\end{array}$} \\
\hline & & $\begin{array}{l}\mathrm{RN} \text { mãe } \\
\text { ausente }\end{array}$ & $\begin{array}{l}\text { RN mãe } \\
\text { HIV }\end{array}$ & $\begin{array}{c}\mathrm{RN} \\
\text { hipoglicemia }\end{array}$ & $\begin{array}{l}\text { RN fator risco } \\
\text { hipoglicemia }\end{array}$ & $\begin{array}{c}\mathrm{RN} \text { mãe } \\
\text { doença grave }\end{array}$ & & \\
\hline \multirow{4}{*}{$\begin{array}{c}\text { Tent } \\
\text { ativa } \\
\text { de } \\
\text { orde } \\
\text { nha }\end{array}$} & $\mathrm{N}$ & 0 & 0 & 7 & 16 & 0 & 23 & \\
\hline & $\%$ & $0 \%$ & $0 \%$ & $17,9 \%$ & $41,0 \%$ & $0 \%$ & $59,0 \%$ & 0,001 \\
\hline & $\mathrm{N}$ & 4 & 3 & 0 & 7 & 2 & 16 & \\
\hline & $\%$ & $10,3 \%$ & $7,7 \%$ &, $0 \%$ & $17,9 \%$ & $5,1 \%$ & $41,0 \%$ & \\
\hline \multirow[t]{2}{*}{ Total } & $\mathrm{N}$ & 4 & 3 & 7 & 23 & 2 & 39 & \\
\hline & $\%$ & $10,3 \%$ & $7,7 \%$ & $17,9 \%$ & $59,0 \%$ & $5,1 \%$ & $100,0 \%$ & \\
\hline
\end{tabular}

De acordo com as indicações da OMS Horizonte em comparação com o estudo para o uso de aleitamento artificial, verificourealizado no HAC no estado do Rio do se maior conformidade do HAC de Belo Janeiro (Tabela 6).

Tabela 6- Comparação dos resultados deste estudo com o estudo prévio realizado em um Hospital Amigo da Criança do estado do Rio de Janeiro.

\begin{tabular}{c|c|c|c}
\hline Amostra & N & Indicações não justificáveis pela OMS & \% \\
\hline Estudo HAC, BH-MG, 2011 & 39 & 4 & 10,25 \\
\hline Estudo HAC, RJ-RJ, 2008* & 300 & 143 & 47,66 \\
\hline
\end{tabular}

*Meirelles et al.

\section{Discussão}

Devido à importância do leite materno $^{18}$, quando não é possível sucção do recém-nascido ao seio da mãe, faz-se necessário a realização de ordenha, com os objetivos de continuar estimulando a lactogênese e evitar o uso de fórmula láctea. Esse procedimento não é feito em mães HIV positivas por ser uma contra-indicação 
absoluta à amamentação ${ }^{19}$. O presente estudo constatou que na maioria dos casos houve a tentativa de ordenha e apenas nos casos em que este procedimento não foi bem sucedido indicou-se o uso de fórmula láctea, de acordo com o que preconiza o IHAC ${ }^{20}$.

O presente estudo constatou que 25 $(64,10 \%)$ recém-nascidos iniciaram o uso de suplementação láctea nas primeiras 12 horas de vida. É importante lembrar que nas primeiras 24 horas de vida, a quantidade de leite materno é pequena e a mama necessita de estímulo, através da sucção. O uso de leite artificial neste período pode abolir os reflexos de busca e sucção do bebê, diminuindo a freqüência das mamadas e o estímulo do mamilo. Além disso, deve-se considerar que recém-nascidos a termo, sem fatores de risco, não apresentam hipoglicemia como resultado de alimentação subótima.

Dos 25 casos mencionados, 8 casos $(32,00 \%)$ iniciaram nas primeiras 2 horas, sendo que 2 casos não houve desejo de amamentar por parte da mãe, demonstrando uma precipitação na prescrição de suplemento lácteo por parte dos profissionais, visto que essa indicação não é preconizada pela $\mathrm{OMS}^{16}$. Tiveram ainda 3 indicações por se tratarem de mães HIV positivas, o que nos confirma a correta indicação feita pelo HAC de Belo Horizonte. Vale ressaltar que esta indicação é considerada absoluta para uso de leite artificial, pois existe uma probabilidade de transmissão vertical pelo HIV, por isso a necessidade de se conhecer o status sorológico da mãe antes de ocorrer o nascimento do recém-nascido. Portanto, o leite artificial só deve ser prescrito em vigência de positividade, pois o aleitamento materno representa um risco adicional de $7,00 \%$ a $22,00 \%^{19}$.

Constatou-se ainda que 2 recémnascidos dos 25 casos iniciaram o uso de leite artificial nas 2 primeiras horas de vida, foram enquadrados pela instituição em estudo com a indicação de risco para hipoglicemia associado à taquipnéia, tendo como conseqüência, a impossibilidade de sucção do recém-nascido devido ao risco de aspiração. Por este motivo, a instituição considera necessário o uso de suplementação láctea, embora a $\mathrm{OMS}^{16}$ não descreva esta situação como uma razão médica aceitável para indicação de leite artificial.

Houve apenas um caso relatado de má formação congênita (fissura labial), o que a instituição justificou para a indicação do uso de fórmula láctea, por gerar uma impossibilidade de sucção. Sabe-se que fissura labial não é contra-indicação para o aleitamento materno, pois uma correta vedação entre o seio da mãe e a fissura labial do recém-nascido, possibilita uma boa sucção. A produção de leite está intimamente ligada ao estímulo pela sucção do recém-nascido, sendo assim, a ausência de estímulo leva a uma redução da quantidade de leite removido das mamas, o que dificulta a ordenha de leite materno. Ressalta-se que essa situação não é descrita pela $\mathrm{OMS}^{16}$. 
Os critérios analisados (idade, escolaridade, estado civil e número de consultas de pré-natal) não interferiram no estudo provavelmente porque as mães se encontravam internadas no alojamento conjunto sendo assistidas e orientadas de acordo com o protocolo do hospital. Embora o desconhecimento materno sobre amamentação possa contribuir para o surgimento de dificuldades na sua prática, levando ao uso indevido de suplemento lácteo $^{5}$.

Do total de justificativas presentes no estudo, a que possui maior prevalência foi o risco para hipoglicemia associado a fatores como, prematuridade, PIG, GIG, quadro infeccioso, sofrimento intraparto com dificuldade de sucção nas primeiras 48 horas e colostro ausente à expressão manual. Isso corresponde à 23 casos $(58,97 \%)$. A prematuridade se justifica pela sucção fisiologicamente débil, dificuldades para deglutir, digerir e absorver, por características anatômicas e neurológicas da idade gestacional $^{21}$. A ineficiência da sucção faz com que a mama seja estimulada de forma inadequada, o que acarreta uma diminuição da produção de leite, deixando os prematuros mais susceptíveis à hipoglicemia, levando ao uso de suplemento ${ }^{22-25}$, e demonstrado no presente estudo.

Segundo a instituição, faz-se necessário o uso de suplemento em recémnascidos, em que a mãe se encontra ausente por qualquer motivo. Entretanto, esta situação especificamente não se encontra nas razões preconizadas pela $\mathrm{OMS}^{16}$, que corresponde a 4 casos $(10,26 \%)$.

Foi utilizado o teste do qui-quadrado por meio do software SPSS na relação entre tentativa de ordenha e indicação do uso de fórmula láctea com significância $(p=0,001)$. Não foram encontrados casos de tentativa de ordenha em mães HIV positivos por ser considerada contraindicação absoluta de acordo com a OMS. Por se tratar de um HAC, observou-se que houve tentativa de ordenha na maioria dos casos antes de se iniciar o uso de fórmula láctea, sendo a prevalência das indicações o risco para hipoglicemia neonatal.

$\mathrm{O}$ estudo de Meirelles et $a l^{25}$, apesar de se tratar de um HAC e da população estudada ser composta de recém-nascidos de baixo risco, um terço deles teve suplemento prescrito e destes, houve uma taxa de $47,66 \%$ (143 casos) com indicações não condizentes com as da OMS. Em contrapartida, o estudo em questão também realizado em um HAC teve uma taxa de 4,54\% (59 casos) de neonatos que fizeram uso de fórmula láctea e destes, apenas 10,25\% (4 casos) tinham indicações que não estavam em conformidade com a OMS. Diante disso, verificou-se que a instituição em estudo atende em sua grande maioria às políticas adotadas pela IHAC em relação ao passo 6 , que preconiza "não oferecer aos recém-nascidos nenhum outro tipo de alimento ou bebida além do leite materno, a menos que exista uma indicação médica". 
O estudo apresentado reforça a já difundida idéia na comunidade científica de que se acumulam as evidências sobre as vantagens do aleitamento materno tanto para o neonato quanto para a mãe.

Embora a OMS tenha indicações bastante criteriosas para o uso de leite artificial, existem situações importantes e freqüentes que não estão enquadradas nelas. Diante disso, os profissionais de saúde responsáveis pela solicitação do uso de suplemento, devem levar em consideração as particularidades do binômio mãe-filho, para que não ocorra o uso indiscriminado da suplementação láctea. Por isso, verificou-se que os profissionais engajados na política do aleitamento materno, devem se basear no protocolo da OMS, mas não deixando de lado seu senso crítico e sensibilidade com condições particulares.

O presente estudo foi importante para verificar se o passo 6 da IHAC estava sendo cumprido no Hospital Sofia Feldman. Diante dos resultados, verificou-se que houve conformidade. Isso torna o hospital estudado, modelo para outras instituições credenciadas à IHAC e exemplo para demais instituições interessadas no credenciamento.

\section{REFERÊNCIAS}

1. Novais JF, Lamounier JA, Franceschini SCC, Priore SE. Efeitos a curto e longo prazo do aleitamento materno na saúde infantil. Rev. Soc. Bras. Aliment. Nutr, 2009; 34:139160.

2. Antunes LS, Antunes LAA, Corvino MPF, Maia LC. Amamentação natural como fonte de prevenção em saúde. Ciência e Saúde Coletiva, 2008; 13: 103-109.

3. Almeida GG de, Spiri WC, Juliani CMCM, Paiva BSR. Proteção, promoção e apoio ao aleitamento materno em um hospital universitário. Ciência e Saúde Coletiva, 2008; 13: 487-494.

4. Balaban G, Silva GAP. Efeito protetor do aleitamento materno contra a obesidade infantil. J. Pediatr., 2004; 80: 7-16.

5. Silva LR. Vieira G, Dias CPF, Santos DRD, Ferraz F, Carneiro G, Casqueiro JB, Boas LV, Oliveira L, Santana MC de, Barreiro P. Conhecimento materno sobre aleitamento: um estudo piloto realizado em Salvador, Bahia visando à elaboração de uma cartilha educativa. Rev. ciênc. méd. biol, 2005; 4:187-194.

6. Sanches MTC, Buccini $\mathrm{G}$ dos $\mathrm{S}$, Gimeno SGA, Rosa TE da C, Bonamigo AW. Fatores associados à interrupção do aleitamento materno exclusivo de lactentes nascidos com baixo peso assistidos na atenção básica. Cad. Saúde Pública, 2011; 27:953-965. 
7. Bunik M. ABM Clinical Protocol \#3: Hospital Guidelines for the Use of Supplementary Feedings in the Healthy Term Breastfed Neonate, Revised 2009 BREASTFEEDING MEDICINE, 2009; 4:175-182.

8. Buchala LM, Moraes MS. Amamentação vivenciada com sucesso por um grupo de mulheres. Arq Ciênc Saúde, 2005; 27: 77-82.

9. Santoro Jr W, Martinez FE, Ricco RG, Gorge SM. Colostrum Ingested during the First Day of Life by Exclusively Breastfed Healthy Newborn Infants. J Pediatr, 2010; 156:29-32.

10. Azeredo CM, Maia $\mathrm{T}$ de $\mathrm{M}$, Rosa TCA, Silva FF, Cecon PR, Cotta RMM. Percepção de mães e profissionais de saúde sobre o aleitamento materno: encontros e desencontros. Rev. paul. Pediatr, 2008; 26: 366-344.

11. Caminha M de FC, Serva VB, dos Anjos MMR, Brito RB de S, Lins MM, Filho, MB. Aleitamento materno exclusivo entre profissionais de um Programa Saúde da Família. Ciênc. saúde coletiva, 2011; 16: 2245-2250.

12. Halpern R, Figueiras ACM. Influências ambientais na saúde mental da criança. Jornal de Pediatria, 2004; 80: 104-110.

13. Toma TS, Rea MF. Benefícios da amamentação para a saúde da mulher e da criança: um ensaio sobre as evidências. Cad. Saúde Pública, 2008; 24:235-246.

14. Marques RFSV, Lopez FA, Braga JAPO. Crescimento de crianças alimentadas com leite materno exclusivo nos primeiros seis meses de vida. J Pediatr, 2004; 80:99-105.

15. Frota MA, da Costa FB, Soares SD, Filho OAS, de Albuquerque C de M, Casimiro CF. Fatores que interferem no aleitamento materno. Rev. RENE, 2009; 10: 61-67.

16. OMS. Razões médicas aceitáveis para uso de substitutos do leite materno, 2009. $276 \mathrm{p}$.

17. Wight N, Marinelli KA. ABM Clinical Protocol \#1: Guidelines for Glucose Monitoring and Treatment of Hypoglycemia in Breastfed Neonates. BREASTFEEDING MEDICINE, 2006; 1:178-184.

18. Boccolini CS, de Carvalho ML, de Oliveira MI, Boccolini $\mathrm{P}$ de $\mathrm{M}$. Breastfeeding can prevent hospitalization for pneumonia among children under 1 year old. J Pediatr, 2011; 87:399-404.

19. Brasília: Ministério da Saúde. Programa Nacional de DST e AIDS, Secretaria de Vigilância em Saúde, Ministério da Saúde. Recomendações para profilaxia da transmissão vertical do HIV e terapia anti-retroviral em gestantes, 2007; 7-145. 
20. Vannuchi MTO, Monteiro CA, Réa MF, Andrade SM de, Matsuo T. Iniciativa Hospital Amigo da Criança e aleitamento materno em unidade de neonatologia. Rev. Saúde Pública, 2004; 38:422-428.

21. Leal DT, do Nascimento L, Fialho FA, Arruda WC, Dias IMAV. O perfil de portadores de diabetes tipo 1 considerando seu histórico de aleitamento materno.Esc. Anna Nery, 2011; 15: 68-74.

22. Araújo MF de M, Otto AFN, Schmitz B de AS. Primeira avaliação do cumprimento dos "Dez Passos para o Sucesso do Aleitamento Materno" nos Hospitais Amigos da Criança do Brasil. Rev. Bras. Saúde Mater. Infant., 2003; 3: 411-19.

23. Lamounier JA. Experiência Iniciativa Hospital Amigo da Criança. Rev. Assoc. Med. Bras., 1998; 44: 319-324.

24. Bernardi JR, Gama CM, Vitolo MR. Impacto de um programa de atualização em alimentação infantil em unidades de saúde na prática do aleitamento materno e na ocorrência de morbidade. Cad. Saúde Pública, 2011; 27: 1213-1222.

25. Meirelles $\mathrm{C}$ de AB, Oliveira MI do C, Mello RR de, Varela MAB, Fonseca $\mathrm{V}$ de $\mathrm{M}$. Justificativas para uso de suplemento em recém-nascidos de baixo risco de um Hospital Amigo da
Criança. Cad. Saúde Pública, 2008;

24:2001-2012.

\section{(1)}

